Rabaska

Revue d'ethnologie de l'Amérique française

\title{
La résistance des marges. Exploration, transfert et revitalisation des traditions populaires
}

\section{Carmen d'Entremont}

Volume 6, 2008

URI : https://id.erudit.org/iderudit/019990ar

DOI : https://doi.org/10.7202/019990ar

Aller au sommaire du numéro

Éditeur(s)

Société québécoise d'ethnologie

ISSN

1703-7433 (imprimé)

1916-7350 (numérique)

Découvrir la revue

Citer ce compte rendu

d'Entremont, C. (2008). Compte rendu de [La résistance des marges.

Exploration, transfert et revitalisation des traditions populaires]. Rabaska, 6,

116-120. https://doi.org/10.7202/019990ar d'utilisation que vous pouvez consulter en ligne. 


\section{Colloques et rassemblements}

\section{La résistance des marges. Exploration, transfert et revitalisation des traditions populaires}

CARMEn D'EnTREMONT

Université Sainte-Anne

Faisant suite à des échanges qui prirent naissance en 1991 et qui ont récemment mené aux premières journées d'études de la Chaire de recherche en oralité des francophonies minoritaires (COFRAM) à la Pointe-de-l'Église en octobre 2005 puis, en juin 2006, aux premières Rencontres britto-franco-canadiennes à Brest, les organisateurs ont convenu d'examiner le phénomène de la Résistance des marges par un colloque international, qui s'est déroulé à 1'Université Sainte-Anne (Nouvelle-Écosse), du 15 au 18 août 2007. Organisé conjointement par la CofRAm et le Groupe de recherche en études acadiennes (GRÉA), avec la collaboration du Centre de recherche bretonne et celtique (CRBC, Université de Bretagne occidentale, Brest), de l'Unité de recherche en médiation culturelle (URMC, Université de Liège) et de la Société québécoise d'ethnologie ( $\mathrm{S}_{\mathrm{QE}}, \mathrm{Québec}$ ), cette rencontre a réuni plus de 80 participants. Une quarantaine de conférenciers réputés dans leur domaine sont venus d'un peu partout en Amérique du Nord, mais aussi de l'Europe et même de l'Afrique pour participer à ce colloque international, qui comprenait quatorze séances. Pendant ces quatre jours, ils abordèrent selon leurs points de vue particuliers le thème de la Résistance des marges en fonction des axes suivants : exploration, transfert et revitalisation des traditions populaires des francophonies d'Europe et d'Amérique.

Dans la conférence d'ouverture, Jean-Pierre Pichette proposa le principe du limaçon, comme métaphore de la résistance culturelle des marges, afin de montrer que la prédilection des ethnologues pour les enquêtes dans des milieux périphériques, plutôt que dans les centres, n'était pas inspirée par un obscur appétit d'exotisme, mais correspondait bien à la réalité des faits. C'est que le colloque avait ciblé les processus de diffusion et de rétention des traditions, les rapports entre le centre et ses périphéries, le rôle des marges, pour tenter de les comprendre. On y aborda l'ancienneté, l'origine plurielle et l'actualité 
des traditions, leur métissage, les facteurs qui ont contribué à leur maintien ou à leur érosion, tant en périphérie qu'au centre. Issus de disciplines multiples (ethnologie, histoire de l'art, linguistique, littérature, folklore, animation culturelle, histoire, anthropologie, archéologie, sociologie, théologie, éducation), les intervenants considérèrent des marges de la francophonie d'horizons divers : tout le Canada français (de l'Acadie à l'Ouest), les ÉtatsUnis (du Wisconsin à la Louisiane) et la Guyane, ainsi que des régions d'Europe (Bretagne, Béarn et Wallonie) et d'Afrique. Le programme comporta encore deux tables rondes et la projection de films ethnographiques. Concrètement, cinq axes fondamentaux actualisaient l'objectif général, balisant la réflexion souhaitée et charpentant le colloque.

\section{L'exploration de la marge : valeur heuristique et construction identitaire}

Un premier axe du colloque porta sur les recherches de terrain dans les régions périphériques ou éloignées, que les premiers « antiquaires 》 ont favorisées. La première séance, qui fut une exploration de la marge en France, renfermait deux communications. Nelly Blanchard, de l'Université de Bretagne Occidentale, confrontant la dialectologie et la standardisation linguistique, fit connaître le rôle très important des centres et marges économiques et culturels en Basse-Bretagne. Jean-François Simon, directeur du CRBC, rappela la place effectivement donnée à la Bretagne dans de nombreuses étapes de la construction du projet scientifique que revendique aujourd'hui l'Ethnologie de la France, et il tenta, ensuite, de lui trouver des éléments d'explication, au rang desquels il considéra l'idée même de marge, associée d'une part à la Bretagne, « à la marge » (de l'espace national), et d'autre part à ses habitants, « en marge » (de la société globale). Ce volet comprenait aussi deux tables rondes : dans la première (séance 7), des chercheurs partagèrent leurs réflexions sur le thème de la marge comme terrain idéal, terrain idéalisé, alors que, dans la deuxième (séance 13), ils discutèrent du rôle des médiateurs culturels dans l'affirmation identitaire.

\section{Les migrations et transferts culturels de l'Europe à l'Amérique}

Ce deuxième volet, distribué en trois séances, portait sur les populations qui ont dû migrer, de gré ou de force, plus particulièrement les Acadiens et les autres Français d'Amérique. Dans un premier temps (séance 3), Jean Simard, de l'Université Laval, analysa les traditions religieuses communes de deux marges : le Québec et la Bretagne catholiques, liés par un même combat de résistance de la marge ethnoreligieuse ; Denise Lamontagne, de l'Université de Moncton, brossant l'histoire de la résistance de la figure de l'aïeule du Christ au sein de l'Église catholique, fit voir comment la sainte d'Auray en Bretagne tout comme celle du Bocage en Acadie nous convient à une relecture 
du concept même de marginalité ; et Pierre-Yves Mocquais, professeur à l'Université de Calgary, analysa les témoignages de François-Marie Rohel, «Breton » de Saint-Brieux, Saskatchewan, qui représentaient en fait le refus d'une « bretonitude » institutionnelle. La séance suivante (séance 6) traita d'une période plus ancienne. Marc Lavoie, de l'Université Sainte-Anne, fit connaître l'origine et la transformation des techniques d'assèchement introduites en Acadie et, plus tard, transmises à la fois chez les Planters des Maritimes et dans les colonies américaines, et il nota la transformation et la survivance de ces techniques (aboiteaux acadiens) à travers le temps. Bernard Cherubini, qui s'intéresse, depuis 1989, aux Acadiens arrivés en Guyane à la suite du désastre de l'expédition de Kourou de 1764, discuta de cette « société d'habitation » qui, structurant des relations autonomes et originales, s'est mise en marge des centres de pouvoir, politiques ou économiques. Enfin, la séance "marge et ethnicité » (séance 11) se composait de deux communications : Françoise Lempereur, de l'Université de Liège, présenta les traditions culturelles des Wallons du Wisconsin (É.-U.), petite communauté « marginale », et discuta de la pertinence de la sauvegarde d'un patrimoine culturel, fondement d'une « ethnicité positive »; et Yves Bergeron, professeur à l'Université du Québec à Montréal, proposa une réflexion sur la crise identitaire observée à travers le processus de patrimonialisation de la culture française en Amérique du Nord (plus particulièrement le territoire culturel du Québec).

\section{Les tensions entre le centre et sa périphérie : les marges, lieux de résistance}

Le troisième axe du colloque comprenait deux séances qui portèrent sur le centre et les marges, lieux de résistance, qui seraient plus conservatrices, plus fidèles à la tradition et plus réfractaires au changement. Dans la séance portant sur la mise en scène de la marge (séance 4), Caroline-Isabelle Caron, de l'Université Queen's, examina le discours historique des fêtes commémoratives en Acadie néo-écossaise au milieu du $\mathrm{Xx}^{\mathrm{e}}$ siècle en tant que discours de la marge ; ensuite, à partir d'une mise en perspective des contenus de dépliants touristiques et pages internet valorisant «les» atouts de la Bretagne et plus particulièrement du Finistère, Marie-Armelle Barbier-Le Déroff, de l'Université de Bretagne Occidentale, interrogea les processus de dynamiques des marges qui s'expriment au travers des catégories de mots et expressions utilisés pour attirer différentes clientèles de touristes; et Linda Guidroux exposa la migration d'un savoir-faire traditionnel en prenant l'exemple de la cuisine des crêpes bretonnes au Québec. L'ambivalence de la marge fit l'objet d'une séance spécifique (séance 5) : Micheline Laliberté, de l'Université Sainte-Anne, analysa les relations et les perceptions entre les Acadiens de la baie Sainte-Marie et les pères eudistes, venus de la France à 
la fin du XIX ${ }^{\mathrm{e}}$ siècle, faisant ressortir les zones de rencontre et de résistance entre ces deux entités ; Pietro Boglioni, de l'Université de Montréal, proposa de vérifier si, et jusqu'à quel point, dans le domaine particulier de la religion populaire canadienne-française, on peut appliquer les mêmes hypothèses aux rapports entre le centre et les marges que l'on applique dans les autres domaines de la culture populaire ; et Ronald Labelle, titulaire de la nouvelle Chaire de recherche McCain en ethnologie acadienne à l'Université de Moncton, illustra les reflets de l'Ankou breton, figure de mort, dans la légende acadienne de la charrette de la mort.

\section{Les ambiguïtés de la marge : idées reçues sur le métissage}

Le quatrième volet du colloque, le plus vaste, s'étala sur cinq séances. L'une fut axée sur une exploration de la marge en Canada (séance 2) : Serge Gauthier, fondateur du Centre de recherche sur l'histoire et le patrimoine de Charlevoix, exposa, par le cas de la région de Charlevoix, l'approche méthodologique du folkloriste canadien Marius Barbeau ; son collègue Christian Harvey, du même organisme, fit voir que la tradition orale de la Route de Sable, milieu dit de culture de pauvreté et totalement délaissé par les folkloristes québécois, est occultée mais très présente et pertinente pour la réflexion ethnologique ; enfin, Laurier Turgeon, professeur à l'Université Laval, s'appuyant sur des exemples tirés du projet d'Inventaire des ressources ethnologiques du patrimoine immatériel (IREPI) du Québec, proposa de considérer cet inventaire comme un moyen efficace de résistance et, plus encore, de revitalisation du patrimoine culturel immatériel des groupes minoritaires. Une séance entière (séance 8) fut consacrée à la Mi-Carême, une tradition qui résiste à la marge : Barbara Le Blanc, de l'Université Sainte-Anne, traitant de la Mi-Carême au Cap-Breton, présenta le masque de ce rituel comme outil de pouvoir dans les marges ; pour sa part, Bérangère Landry, qui a œuvré dans l'enseignement, exposa la fonction sociale de la Mi-Carême à Natashquan en 2007, tandis que Georges Arsenault, ethnologue indépendant de Charlottetown, décrivit une tradition française moins connue qui a survécu chez les Acadiens de l'Île-du-Prince-Édouard : la Mi-Carême, distributrice de friandises aux petits enfants sages. Dans une troisième séance (séance 9), intitulée «marginalité en musique : inventer sans renier », Barry-Jean Ancelet, de l'Université de la Louisiane à Lafayette, discuta de l'évolution de la musique cadienne, surtout des divers changements stylistiques qui sont dus à la créativité des musiciens et chanteurs ou liés à l'évolution du contexte musical ; pour sa part, Lisa Ornstein, ethnomusicologue intéressée à la musique instrumentale traditionnelle, proposa un regard sur la dynamique de la persistance dans le milieu de la musique et de la danse traditionnelle ; et Pierre Chartrand, spécialiste de la danse ancienne et professeur au cégep de Lanaudière, traita 
de la gigue québécoise, tributaire de la danse irlandaise et écossaise. Enfin, les deux dernières séances de cette section (séance 10 et séance 12) furent consacrées à la marge et à l'identité. À partir des travaux de Michel Foucault, Denis Gagnon, titulaire de la chaire de recherche du Canada sur l'identité métisse, analysa les revendications identitaires récentes des Métis canadiens en mettant l'accent sur les relations de pouvoir entre les « vrais » Métis et les « autres » Métis canadiens, tandis que Patrice Kouraogo, de l'Université Senghor, traitant des migrations des peuples africains en Europe, illustra les transferts des habitudes culturelles par le cas des Burkinabé de France. Marcel Bénéteau, de l'Université de Sudbury, présenta les caractéristiques du répertoire de chansons traditionnelles françaises du Détroit, terrain de marge par excellence, et l'éclairage qu'il apporte à l'étude de la chanson traditionnelle française en Amérique française ; Ariane Bruneton, de l'Université de Pau et des pays de l'Adour, examina les transferts culturels du Béarn aux Amériques ; Éva Guillorel, de l'Université de Rennes 2, analysa le traitement des héros dans la chanson en langue bretonne, une expression de la résistance de la marge face au discours du centre ; et enfin, Robert Bouthillier, directeur du Conseil québécois du patrimoine vivant, apprécia la nature et la constitution du répertoire chanté des francophones d'Amérique, tout spécialement de la communauté acadienne du nord-est du Nouveau-Brunswick, en faisant ressortir les éléments du fonctionnement de la tradition orale qui témoignent du niveau de cette résistance.

\section{La revanche des marges}

Le cinquième axe du colloque (séance 14) porta sur la revivification de la marge, présentement à la mode. Fañch Postic, directeur du Centre de recherche et de documentation sur la littérature orale, antenne du CRBC, scruta les avatars de la quête de l'« eginane "; après une réflexion sur l'origine, le sens et la fonction de la quête, son intervention s'attacha à analyser son évolution ou sa disparition en Europe, et son adaptation et sa diffusion dans les minorités franco-américaines sous la forme de la guignolée. Enfin, Donatien Laurent, de l'Université de Bretagne occidentale, dont les travaux et les échanges avec l'organisateur principal ont inspiré la tenue de ce colloque, traita de la revivification des œuvres dans les marges ; utilisant l'exemple de la chanson du Roi Renaud, « pièce maîtresse du répertoire populaire de langue française », il montra comment une culture orale, particulièrement celle de la Bretagne située en marge, continue à se nourrir de l'œuvre et à la transmettre tant qu'elle conserve sens et valeur.

Ce colloque international abordait des questions d'actualité, soutenues par des propos riches et variés sur lesquels on voudra encore réfléchir. On pourra sans doute le faire prochainement quand paraitront les actes de ce colloque annoncés pour l'automne 2008. 